\title{
Differential Diagnosis of Human Sparganosis Using Multiplex PCR
}

\author{
Hyeong-Kyu Jeon', Kyu-Heon Kim², Woon-Mok Sohn ${ }^{3}$, Keeseon S. Eom ${ }^{1, *}$ \\ ${ }^{1}$ Department of Parasitology, Parasite Research Center and Parasite Resource Bank, Chungbuk National University School of Medicine, Cheongju \\ 28644, Korea; ' $M$ Ministry of Food and Drug Safety, Cheongju 28159, Korea; ${ }^{3}$ Department of Parasitology and Tropical Medicine, Institute of Health \\ Sciences, Gyeongsang National University College of Medicine, Jinju 52727, Korea
}

\begin{abstract}
Human sparganosis was diagnosed by morphological and genetic analyses in Korea. The complete mitochondrial genomes of Spirometra erinaceieuropaei and S. decipiens isolated in Korea have been recorded. Present study was performed to provide information to diagnose the etiologic agent of sparganosis by multiplex PCR using mitochondrial genome sequences of $S$. erinaceieuropaei and $S$. decipiens. In an effort to examine the differential diagnosis of spirometrid tapeworms, multiplex PCR assays were performed on plerocercoid larvae of S. erinaceieuropaei and S. decipiens. The PCR products obtained using species-specific primers were positively detected in all PCR assays on mixture of $S$. erinaceieuropaei and S. decipiens DNA. S. erinaceieuropaei-specific bands (239 bp and $401 \mathrm{bp}$ ) were obtained from all PCR assays using a mixture of S. erinaceieuropaei-specific primers (Se/Sd-1800F and Se-2018R; Se/Sd-7955F and Se8356R) and S. erinaceieuropaei template DNA. S. decipiens-specific bands (540 bp and 644 bp) were also detected in all PCR assays containing mixtures of S. decipiens-specific primers (Se/Sd-1800F and Sd-2317R; Se/Sd-7955F and Sd8567R) and S. decipiens template DNA. Sequence analyses on these species-specific bands revealed $100 \%$ sequence identity with homologous regions of the mtDNA sequences of $S$. erinaceieuropaei and $S$. decipiens. The multiplex PCR assay was useful for differential diagnosis of human sparganosis by detecting different sizes in species-specific bands.
\end{abstract}

Key words: Spirometra erinaceieuropaei, Spirometra decipiens, differential diagnosis, multiplex PCR, human sparganosis

Human sparganosis is an infection caused by the plerocercoid larvae of the genus Spirometra, a kind of diphyllobothriidean tapeworms. These parasites require 2 different intermediate hosts: the fresh water copepods as the first intermediate host, and vertebrates such as amphibians, reptiles and mammals as the paratenic host. Humans become infected through ingestion of these intermediate hosts. Spirometra erinaceieuropaei is the most well-known as the type species in the genus Spirometra (Cestoda: Diphyllobothriidae) [1-4]. The plerocercoid larvae of spirometrid tapeworm (spargana) invade the brain, eyes, breast, spinal cord, and subcutaneous tissue of humans $[5,6]$. Sparganosis is a parasitic zoonosis that can cause serious clinical problems in human according to the invasive organs. More than 1,600 cases have been reported in the world. Most cases occurred in East and Southeast Asia, and sporadically in

\footnotetext{
- Received 18 February 2018, revised 3 April 2018, accepted 20 April 2018.

*Corresponding author (kseom@chungbuk.ac.kr)

(C) 2018, Korean Society for Parasitology and Tropical Medicine

This is an Open Access article distributed under the terms of the Creative Commons Attribution Non-Commercial License (http://creativecommons.org/licenses/by-nc/4.0) which permits unrestricted non-commercial use, distribution, and reproduction in any medium, provided the original work is properly cited.
}

South America, Europe and Africa [7]. The precise identification of these spirometrid tapeworms is important in efforts to control diseases that lead to human sparganosis.

Molecular identification has played an important role over the decades in studies pertaining to phylogenetics, parasite genetic variation and evolution. Mitochondrial DNA sequences have provided genetic markers for phylogenetic reconstruction, taxonomic identification, population genetics, and epidemiological investigations [8]. DNA sequencing of PCR products can be employed for molecular differential diagnosis and identification of Spirometra species using DNA sequence analysis of small (18S) and large (28S) subunit ribosomal RNA, ribosomal internal transcribed spacer 1, ribosomal internal transcribed 2, and mitochondrial genes [9-11]. Investigations of the phylogenetic relationships and genetic variations of Spirometra tapeworms have utilized mitochondrial genomes or genes such as cytochrome $c$ oxidase subunit $1(\operatorname{cox} 1)$ and 3 (cox3) and NADH dehydrogenase subunit 1 (nad1), 3 (nad3), and 4 (nad4) [12-17].

Recently, Spirometra species inducing human sparganosis in Korea have been identified by morphological and mitochon- 
drial cox 1 analysis [18], and the complete mitochondrial genomes of S. erinaceieuropaei and S. decipiens, which cause human sparganosis, have been sequenced and analyzed [19]. These reports in Korea have included Spirometra decipiens as a possible causative agent for causing human sparganosis in addition to $S$. erinaceieuropaei, the latter being a well-known single parasitic agent for human sparganosis. Therefore, a rapid and sensitive method is required for unambiguous species identification with differential diagnosis of human sparganosis. A reliable method for the identification of human infecting Spirometra tapeworms is necessary to assist with surveillance, disease control, and improve our understanding of the epidemiology of this foodborne zoonosis. In particular, questions remain concerning $S$. erinaceieuropaei and its infection route (waterborne vs foodborne) in humans, and where in a recent paper on this parasite questions concerning the correct intermediate and final host animals in their life cycle were not addressed [18].

The aim of this study was to develop a high-resolution multiplex PCR assay using species-specific primers based on the mitochondrial sequences of $S$. erinaceieuropaei and $S$. decipiens to distinguish between $S$. erinaceieuropaei and $S$. decipiens that can be utilized for the differential diagnosis of human sparganosis from other cestodes.

A total of 25 parasites were used in this study, comprising 8 plerocercoids of $S$. erinaceieuropaei, 7 plerocercoids of $S$. decipiens from infected humans in Korea, and negative control samples comprising Taenia asiatica $(\mathrm{n}=1)$, T. saginata $(\mathrm{n}=1)$, T. pisiformis $(\mathrm{n}=1), T$. hydatigena $(\mathrm{n}=1)$, T. crassiceps $(\mathrm{n}=1), H y$ datigera taeniaeformis [18] $(\mathrm{n}=1)$, Echinococcus multilocularis $(\mathrm{n}=1)$, Diphyllobothrium latum $(\mathrm{n}=1)$, and D. nihonkaiense $(\mathrm{n}=1)$. For molecular characterization, all materials employed were identified by sequencing of the mitochondrial cox1 gene.

Two sets of species-specific primers were designed to generate species-specific PCR products of different size based on the nucleotide sequences of cytochrome $b(c o b)$, NADH dehydrogenase subunit 4L (nad4L), NADH dehydrogenase subunit 4 (nad4), cytochrome $c$ oxidase subunit 1 (cox1), and large subunit ribosomal DNA (lrDNA) from S. erinaceieuropaei and S. decipiens. The forward primer was designed from a highly conserved region in cob gene, Se/Sd-1800F (5'-AGTTATTTTCGGTTGGTGCTGTAG-3'; nucleotides 1799 to 1822 from KJ599679 and nucleotides 1800 to 1823 from KJ599680). The 2 reverse primers were designed to amplify products of different length as follows: Se2018R, specific for S. erinaceieuropaei (5'-CCACAAACCCAATAACAAACTA-3'; nucleotides 2018 to 2039 from KJ599680); and Sd-2317R, specific for S. decipiens (5'-TCCTCCCCCACACGACAAAA-3'); nucleotides 2317 to 2337 from KJ599679.

The forward primer, Se/Sd-7955F (5'- ACGTGGTTTGTG GTGGCTCATTTT-3'; nucleotides 7946 to 7969 from KJ599679 and nucleotides 7955 to 7978 from KJ599680), was from a highly conserved sequence common to both Spirometra tapeworms. The 2 species-specific reverse primers were designed from different positions of the cox 1 by mitochondrial by mitochondrial and lrDNA regions as follows: Se-8356R, specific for S. erinaceieuropaei (5'-ATGATAGGGTATAGGTGACCA-3'; nucleotides 8336 to 8356 from KJ588680), and Sd-8567R, specific for $S$. decipiens (5'- TTATTAACTTCCTAACCAACTTGATAC-3'; nucleotides 8567 to 8593 from KJ599679) (Table 1).

PCR assays were performed in $25 \mu \mathrm{l}$ reaction mixtures comprising $100 \mathrm{ng}$ of genomic DNA of each species, $10 \mathrm{pmol}$ of each primer, $10 \times$ buffer, $10 \mathrm{mM}$ dNTP mix, $25 \mathrm{mM} \mathrm{MgCl}_{2}$, and 2.5 unit Taq polymerase (High Fidelity PCR system, Roche, Mannheim, Germany). The multiplex PCR protocol consisted of 35 cycles of predenaturation ( $3 \mathrm{~min}$ at $94^{\circ} \mathrm{C}$ ), denaturation (30 sec at $94^{\circ} \mathrm{C}$ ), annealing $\left(40 \sec\right.$ at $\left.45^{\circ} \mathrm{C}\right)$, and extension (60 sec at $72^{\circ} \mathrm{C}$ ), followed by 1 cycle of $5 \mathrm{~min}$ at $72^{\circ} \mathrm{C}$.

PCR products on human sparganum DNA samples using species-specific primers and multiplex PCR assay are presented in Fig. 1. S. erinaceieuropaei-specific bands (239 bp and 401

Table 1. Sequence and position of PCR primers used in present study

\begin{tabular}{lll}
\hline Primer & \multicolumn{1}{c}{ Primer sequence $\left(5^{\prime}\right.$-3') } & Position in mitochondria gene \\
\hline Se/Sd-1800F & TAT TT CGG TTG GTG CTG TAG & cob (Sd, 1799-1822; Se, 1800-1823) \\
Se-2018R & CCA CAA ACC CAA TAA CAA ACT A' & nad4L (Se, 2018-2039) \\
Sd-2317R & TCC TCC CCC CAC ACG ACA AAA & nad4 (Sd, 2317-2337) \\
Se/Sd-7955F & ACG TGG TT GTG GTG GCT CAT Tा' & cox1 (Sd, 7946-7969; Se, 7955-7978) \\
Se-8356R & ATG ATA GGG TAT AGG TGA CCA & IrDNA (Se, 8336-8356) \\
Sd-8567R & TTA TTA ACT TCC TAA CCA ACT TGA TAC & IrDNA (Sd, 8567-8593) \\
\hline
\end{tabular}

Se, S. erinaceieuropaei; Sd, S. decipiens. 
bp) were obtained from all PCR amplifications using a mixture of S. erinaceieuropaei-specific primers (Se/Sd-1800F and Se-2018R; Se/Sd-7955F and Se-8356R) and S. erinaceieuropaei DNA (Fig. 1, lanes 1 and 6). S. decipiens-specific bands (540 bp and 644 bp) were also detected in all PCR assays contain-

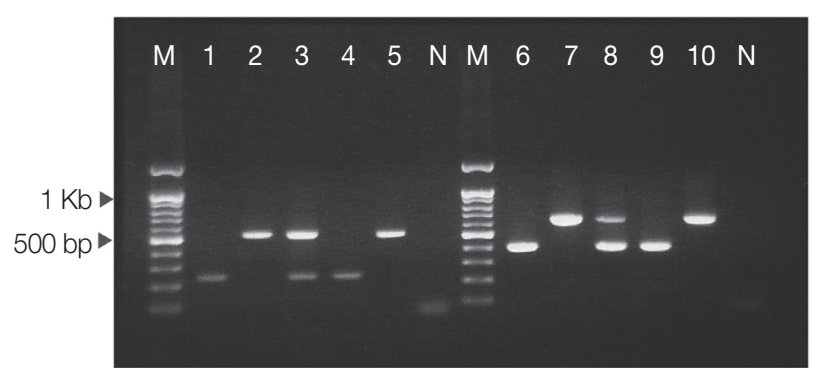

Fig. 1. Multiplex PCR performed with species-specific primer sets. Lanes 1 and 6, S. erinaceieuropaei genomic DNA; lanes 2 and $7, S$. decipiens genomic DNA; lanes 3 and 8 , a mixture of $S$. erinaceieuropaei and S. decipiens genomic DNAs; lanes 4 and 9 , PCR reaction with $S$. erinaceieuropaei-specific primers and a mixture of $S$. erinaceieuropaei and $S$. decipiens genomic DNAs; lanes 5 and 10, PCR reaction with $S$. decipiens-specific primers and a mixture of $S$. erinaceieuropaei and $S$. decipiens genomic DNAs; lane M, DNA size marker (100 bp ladder); N, negative control (no template). ing mixtures of $S$. decipiens-specific primers (Se/Sd-1800F and Sd-2317R; Se/Sd-7955F and Sd-8567R) and S. decipiens template DNA (Fig. 1, lanes 2 and 7). Specificity of the PCR products was confirmed by sequencing. In an effort to differentially diagnose Spirometra plerocercoid infections using a single test, multiplex PCR assays were performed using a mixture of all primers and genomic DNA of Spirometra plerocercoid. Two sets of species-specific primers (Se/Sd-1800F and Se-2018R; Se/Sd-1800F and Sd-2317R) were combined in a single PCR assays using a mixture of $S$. erinaceieuropaei and $S$. decipiens template DNA, which generated $239 \mathrm{bp}$ and $540 \mathrm{bp}$ band fragments (Fig. 1, lane 3). Another set of species-specific primers (Se/Sd-7955F and Se-8356R; Se/Sd-7955F and Sd-8567R) was combined in a single PCR assay using a mixture of $S$. erinaceieuropaei and $S$. decipiens template DNA, which generated PCR products comprising 401 bp and 644 bp fragments (Fig. 1 , lane 8 ). In an effort to examine the sensitivity of the speciesspecific primers, 2 sets of primers (Se/Sd-1800F and Se-2018R; Se/Sd-7955F and Se-8356R) were combined in a single PCR reaction containing S. erinaceieuropaei DNA only (Fig. 1, lanes 4 and 9), and another 2 sets of primers (Se/Sd-1800F and Sd2317R; Se/Sd-7955F and Sd-8567R) were used in a single PCR
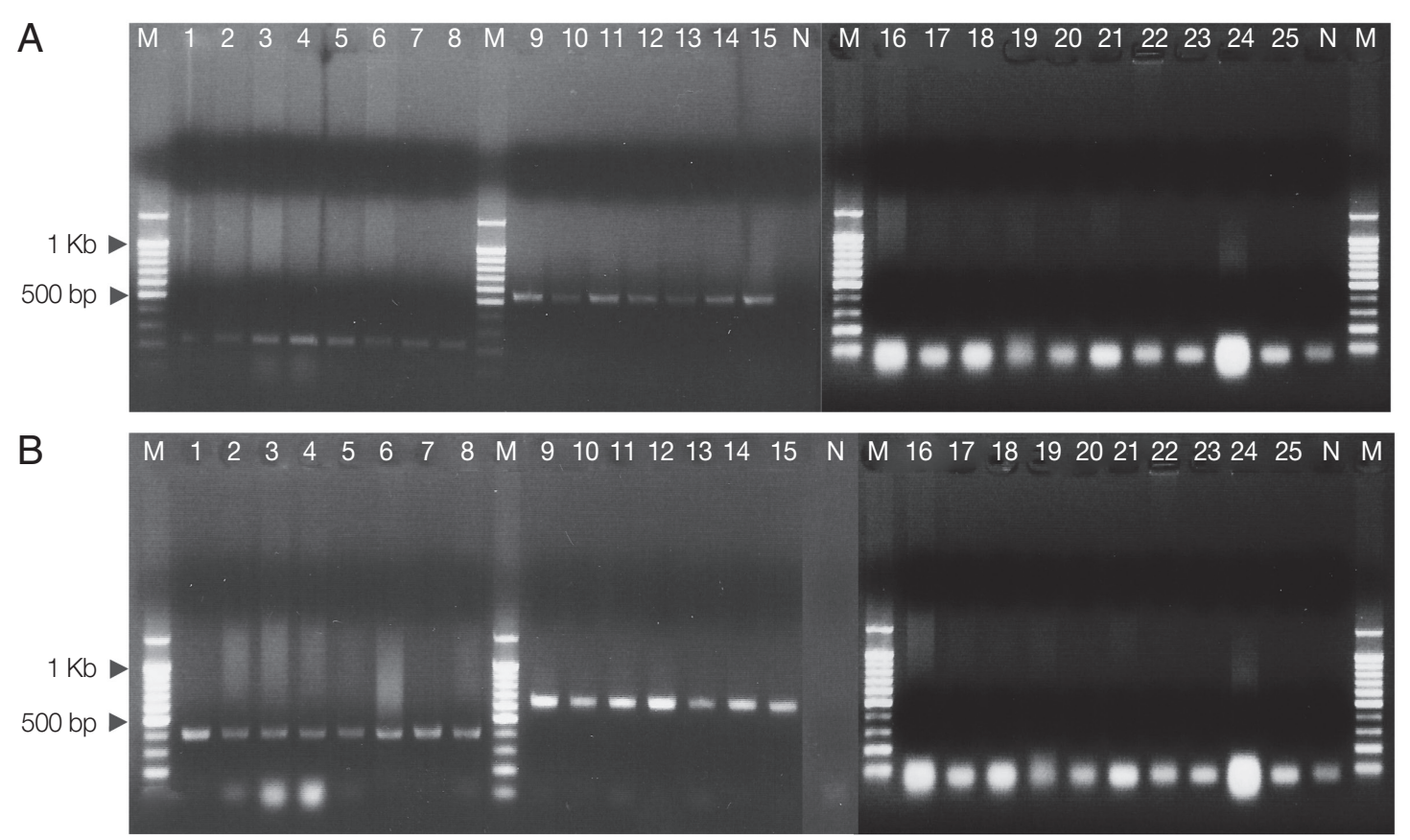

Fig. 2. Differential diagnosis of Spirometra tapeworms by multiplex PCR. (A) S. erinaceieuropaei (lanes 1-8, 239 bp); S. decipiens (lanes 9-15, 540 bp). (B) S. erinaceieuropaei (lanes 1-8, 401 bp); S. decipiens (lanes 9-15, 644 bp). Lane M, DNA size markers (100 bp ladder); Taenia asiatica (lane 16); T. saginata (lane 17); T. solium (lane 18); Hydatigera taeniaeformis (lane 19); T. pisiformis (lane 20); T. hydatigena (lane 21); T. crassiceps (lane 22); Echinococcus multilocularis (lane 23); Diphyllobothrium latum (lane 24); D. nihonkaiense (lane 25); N, Negative control (no template). 
reaction containing $S$. decipiens template DNA only (Fig. 1, lanes 5 and 10).

To detect Spirometra plerocercoid infections, multiplex PCR assays were established utilizing DNA samples prepared from 15 cases of human sparganosis. Diagnostic bands, $239 \mathrm{bp}$ and $401 \mathrm{bp}$, were produced by multiplex PCR of $S$. erinaceieuropaei plerocercoid from human sparganosis (Fig. 2A, lanes 1-8; Fig. 2B, lanes 1-8), while $540 \mathrm{bp}$ and 644 bp diagnostic bands were detected in the plerocercoid of $S$. decipiens from human sparganosis (Fig. 2A, lanes 9-15; Fig. 2B, lanes 9-15). No crossreaction was detected with $S$. erinaceieuropaei- or $S$. decipiensspecific primers when other genomic DNA was employed from human infecting tapeworms such as Taenia, Echinococcus, Hydatigera, and Diphyllobothrium template DNAs (Fig 2A, B, lanes 16-25). No products were amplified from negative control samples by the multiplex PCR assay.

Since the first Korean sparganosis case described in 1954, human sparganosis has been reported in Korea in as many as 134 cases, involving cerebrospinal, subcutaneous, visceral, ocular, testicular, intramuscular, breast or multiple sparganosis. More than 1,600 cases of human sparganosis have been reported worldwide, with more than $80 \%$ of these being from China, Korea, Thailand, Japan, and the USA [7]. Although this genus comprises many species, a consensus on the genus Spirometra has not been achieved with respect to clear species classification. Many epidemiological questions pertaining to these parasites remain concerning species validity, geographical distribution, and transfer or final hosts. More recently, $S$. erinaceieuropaei and $S$. decipiens have been identified from human sparganosis by morphological and genetic analysis in Korea [18]. These findings show that more than 2 Spirometra species are involved in human sparganosis in Korea, therefore, it is required to identify 2 Spirometra species of a causative agents for inducing human sparganosis. Differential identification of plerocercoids of the Spirometra species on the basis of morphological characters alone is problematic. Moreover, correct discrimination of plerocercoids of the Spirometra species obtained from various intermediate hosts using morphology has been considered nearly impractical. Molecular tools utilizing mitochondrial or nuclear genes have proven to be of diagnostic significance in the case of species of intermediate hosts that are related to human infections. Genetic markers could be useful in clarifying epidemiological, ecological, and geographical questions concerning Spirometra tapeworms. Differential diagnosis of the plerocercoids of S. erinaceieuropaei and S. decipiens, in addition to other cestodes, requires the establishment of molecular diagnostic techniques. A PCR-based molecular assay can be useful for the rapid and accurate differentiation of $S$. erinaceieuropaei and $S$. decipiens, in addition to other cestode parasites.

Controversies remain concerning the species classification of tapeworms belonging to the genus Spirometra. The genus Spirometra has been studied intensively by many researchers since 1929 and a variety of spirometrid species have been identified [20-22]. Six Spirometra species were examined morphologically for species identification [20]. The S. mansonoides was identified from cats in the Syracuse region (USA) in 1937 [21]. Fourteen Spirometra species were recognized as being composed of 2 separate groups, the 'bresslauei' and 'okumurai' [22]. All species in the genus Spirometra were synonyms of $S$. erinaceieuropaei [1]. Following extensive experimental studies of morphological variations, with particular emphasis on uterine coils, Iwata (1972) concluded that the morphological difference in uterine shape of the mature proglottids of S. erinaceieuropaei was caused by differences in the developmental larval stages [3]. S. erinaceieuropaei, S. pretoriensis, $S$. theileri and $S$. mansonoides were the only valid species designated by Kamo (1999) [2]. All Spirometra species found in Asian countries were considered synonymous with S. erinaceiruropaei [1-3]. Approximately 38 species of the genus Spirometra have been reported from intestinal parasites of wild and domesticated carnivores $[1-3,20,27]$. Recent molecular data of these spirometrids suggests it includes many species of Spirometra from Asian, South American, and African isolates. Thirty-seven human sparganosis were reported from West African countries 34 specimens from South Sudan and 3 specimens from Ethiopia [23]. These human sparganosis was unclear and not be fully understood which Spirometra species related with human infections by mitochondrial cox 1 sequences analyzed. In Afri$\mathrm{ca}$, the final hosts of Spirometra species were noted wild carnivores such as hyenas and lions. The parasite in the hyenas is known to be $S$. pretoriensis and that from the lions $S$. theileri [24-26]. The range of Spirometra species in Africa is unclear and not known relative role for wide host susceptibility in intermediate and paratenic hosts. The South America isolates of Spirometra spp. were reported from Brazilian isolates that revealed neither $S$. erinaceieuropaei nor $S$. decipiens by mitochondrial cox 1 sequence analyzed. The partial cox 1 nucleotide divergence of Asian isolate identified as $S$. decipiens was differed from South America isolates (17.8-19.2\%) while those of S. erincei- 
europaei (KJ599680) were differed 12.2-12.9\% [27]. Those cases were differed from Asian isolates of $S$. erinaceieuropaei and $S$. decipiens as well as those of African countries by analysis of mitochondrial DNA sequence data. These differences of taxonomic and phylogenetic results provoked epidemiological some questions concerning what is the geographical distribution of Spirometra spp. in worldwide? and which Spirometra species exists South America, Asian, and African countries? These inconsistencies suggest further studies are needed to better understand the taxonomic status of Spirometra spp.

In the present study, we developed a credible multiplex PCR assay for the differential identification of human infecting Spirometra species. The species-specific primers utilized in this multiplex PCR assay comprised 4 sets of species-specific primers that can generate PCR amplicons of different size from spirometrid parasites. The forward primers were derived from highly conserved sequences in the mitochondrial $c o b$ and $c o x 1$ genes, and species-specific reverse primers were designed from highly unrelated sequences in nad4L, nad4 and large subunit ribosomal DNA from both Spirometra tapeworms. This multiplex PCR assay is effective for differential diagnosis in the case of eggs, larva or proglottid, and has a detection sensitivity consisting of a minimum concentration of 50 eggs per gram from a fecal sample (data not shown). These fecal samples were stored in $0.85 \%$ saline or $70 \%$ ethanol at $20^{\circ} \mathrm{C}$ until use. Use of this multiplex PCR assay allows for differential identification of eggs, larva and adult worms, can assist in tracing the origin of the infective plerocercoids present in intermediate hosts, and can help to identify new intermediate hosts in addition to final host animals.

In conclusion, the results of the present study demonstrated that a multiplex PCR assay utilizing mtDNA sequences can be an effective and reliable molecular tool for the identification of Spirometra species causing human sparganosis. The multiplex PCR assay utilizing Se/Sd-1800F, Se-2018R, Se/Sd-7955F and Se-8356R primers for S. erinaceieuropaei, and Se/Sd-1800F, Sd-2317R, Se/Sd-7955F and Sd-8567R primers for S. decipiens should be useful in the differential diagnosis, molecular characterization, and epidemiological survey of human sparganosis by $S$. erinaceieuropaei and S. decipiens.

\section{ACKNOWLEDGMENTS}

This work was supported by the National Research Foundation of Korea (2014R1A1A2004933). Parasite materials used in this study were provided by the Parasite Resource Bank of Korea National Research Resource Center (2010-0003456), the Republic of Korea.

\section{CONFLICT OF INTEREST}

We have no conflict of interest related to this work.

\section{REFERENCES}

1. Yamaguti S. Systema Helminthum. Vol. II. The cestodes of vetebrates. New York, USA. Interscience Publishers. 1959, pp 358361.

2. Kamo H. Guide to Identification of Diphyllobothriid Cestodes. Tokyo, Japan. Gendai Kikaku. 1999, pp 146 (in Japanese).

3. Iwata S. Experimental and morphological studies of Manson's tapeworm, Diphyllobothrium erinacei, Rudolphi. Special reference with its scientific name and relationship with Sparganum proliferum, Ijima. Progr Med Parasitol Jpn 1972; 4: 536-590.

4. Okamoto M, Iseto S, Shibahara T, Sato MO, Wandra T, Craig PS, Ito A. Intraspecific variation of Spirometra erinaceieuropaei and phylogenetic relationship between Spirometra and Diphyllobothrium inferred from mitochondrial CO1 gene sequence. Parasitol Int 2007; 56: 235-238.

5. Cho SY, Bae JH, Seo BS. Some aspects of human sparganosis in Korea. Korean J Parasitol 1975; 13: 60-77.

6. Boonyasiri A, Cheunsuchon P, Suputtamongkol Y, Yamasaki H, Sanpool O, Maleewong W, Intapan PM. Nine human sparganosis cases in Thailand with molecular identification of causative parasite species. Am J Trop Med Hyg 2014; 51: 389-393.

7. Liu Q, Li MW, Wang ZD, Zhao GH, Zhu XQ. Human sparganosis, a neglected food borne zoonosis. Lancet Infect Dis 2015; 15: 1226-1235.

8. Boore JL. Animal mitochondrial genomes. Nucleic Acids Res 1999; 27: 1767-1780.

9. Miyadera H, Kokaze A, Kuramochi T, Kita K, Machinami R, Noya O, Alarcón de Noya B, Okamoto M, Kojima S. Phylogenetic identification of Sparganum proliferum as a pseudophyllidean cestode by the sequence analyses on mitochondrial COI and nuclear sdhB genes. Parasitol Int 2001; 50: 93-104.

10. Liu W, Liu GH, Li F, He DS, Wang T, Sheng XF, Zeng DL, Yang FF, Liu Y. Sequence variability in three mitochondrial DNA regions of Spirometra erinaceieuropaei spargana of human and animal health significance. J Helminthol 2012; 86: 271-275.

11. Dai RS, Liu GH, Song HQ, Lin RQ, Yuan ZG, Li MW, Huang SY, Liu W, Zhu XQ. Sequence variability in two mitochondrial DNA regions and internal transcribed spacer among three cestodes infecting animals and humans from China. J Helminthol 2012; 86: 245-251.

12. Boonyasiri A, Cheunsuchon $P$, Srirabheebhat P, Yamasaki H, Maleewong W, Intapan PM. Sparganosis presenting as cauda 
equina syndrome with molecular identification of the parasite in tissue sections. Korean J Parasitol 2013; 51: 739-742.

13. Zhu XQ, Beveridge I, Berger L, Barton D, Gasser RB. Singlestrand conformation polymorphism-based analysis reveals genetic variation within Spirometra erinacei (Cestoda: Pseudophyllidea) from Australia. Mol Cell Probes 2002; 16: 159-165.

14. Liu W, Zhao GH, Tan MY, Zeng DL, Wang KZ, Yuan ZG, Lin RQ, Zhu XQ, Liu Y. Survey of Spirometra erinaceieuropaei spargana infection in the frog Rana nigromaculata of the Hunan Province of China. Vet Parasitol 2010; 173: 152-156.

15. Zhang X, Cui J, Wei T, Li LY, Jiang J, Lu JC, Jiang P, Liu LN, Wang ZQ. Survey and genetic variation of Spirometra erinaceieuropaei sparganum in frogs and snakes from Guangxi of southern China. Trop Biomed 2014; 31: 862-870.

16. Zhang X, Wang H, Cui J, Jiang P, Fu GM, Zhong K, Zhang ZF, Wang ZQ. Characterisation of the relationship between Spirometra erinaceieuropaei and Diphyllobothrium species using complete $c y t b$ and cox 1 genes. Infect Genet Evol 2015; 35: 1-8.

17. Zhang X, Wnag H, Cui J, Jiang P, Lin ML, Zhang YL, Liu RD, Wang ZQ. The phylogenetic diversity of Spirometra erinaceieuropaei isolates from southwest China revealed by multi genes. Acta Trop 2016; 156: 108-114.

18. Jeon HK, Park HS, Lee DM, Choe SJ, Kim KH, Huh S, Sohn WM, Chai JY, Eom KS. Human infections with Spirometra decipiens plerocercoids identified by morphologic and genetic analyses in Korea. Korean J Parasitol 2015; 53: 299-305.

19. Eom KS, Park HS, Lee DM, Choe SJ, Kim KH, Jeon HK. Mitochondrial genome sequences of Spirometra erinaceieuropaei and $S$. decipiens (Cestoidea: Diphyllobothriidae). Korean J Parasitol 2015; 53: 455-463.

20. Faust EC, Campbell HE, Kellogg CR. Morphological and biological studies on the species of Diphyllobothrium in China. Am J Hyg 1929; 9: 560-583.

21. Mueller JF. New host records for Diphyllobothrium mansonoides Mueller, 1935. J Parasitol 1937; 23: 313-315.

22. Wardle RA, McLeod JA. The Zoology of Tapeworms. Minneapolis, USA. Minnesota University Press. 1952, pp 559-615.

23. Eberhard ML, Thiele EA, Yembo GE, Yibi MS, Cama VA, Tiben ER. Thirty-seven human cases of sparganosis from Ethiopia and South Sudan caused by Spirometra spp. Am J Trop Med Hyg 2015; 93: 350-355.

24. Berensten AR, Becker MS, Stockdale-Walden H, Matandiko W, McRobb R, Dunbar MR. Survey of gastrointestinal parasite infection in African lion (Panthera leo), African wild dog (Lycaon pictus) and spotted hyaena (Crocuta crocuta) in the Luangwa Valley, Zambia. Afr Zool 2012; 47: 363-368.

25. Müller-Graf CD. A coprological survey of intestinal parasites of wild lions (Panthera leo) in the Serengeti and the Ngorongoro Crater, Tanzania, East Africa. J Parasitol 1995; 81: 812-814.

26. Müller-Graf CD, Woolhouse ME, Packer C. Epidemiology of an intestinal parasite (Spirometra spp.) in two populations of African lions (Panthera leo). Parasitology 1999; 118: 407-415.

27. Almeida GG, Coscarelli D, Melo MN, Melo AL, Pinto HA. Molecular identification of Spirometra spp. (Cestoda: Diphyllobothiidae) in some wild animals from Brazil. Parasitol Int 2016; 65: 428-431. 